\title{
Generation and development of polarization speckle based on random walks of polarization phasor
}

Wang, Wei; Lee, Tim K.; Hanson, Steen Grüner; Takeda, Mitsuo

Published in:

Proceedings of SPIE

Link to article, DOI:

$10.1117 / 12.2523353$

Publication date:

2019

Document Version

Publisher's PDF, also known as Version of record

Link back to DTU Orbit

Citation (APA):

Wang, W., Lee, T. K., Hanson, S. G., \& Takeda, M. (2019). Generation and development of polarization speckle based on random walks of polarization phasor. In Proceedings of SPIE (Vol. 11052). [110521N] SPIE International Society for Optical Engineering. Proceedings of SPIE - The International Society for Optical Engineering https://doi.org/10.1117/12.2523353

\section{General rights}

Copyright and moral rights for the publications made accessible in the public portal are retained by the authors and/or other copyright owners and it is a condition of accessing publications that users recognise and abide by the legal requirements associated with these rights.

- Users may download and print one copy of any publication from the public portal for the purpose of private study or research.

- You may not further distribute the material or use it for any profit-making activity or commercial gain

- You may freely distribute the URL identifying the publication in the public portal 


\section{Generation and development of polarization speckle based on random walks of polarization phasor}

Wei Wang, Tim K. Lee, Steen G. Hanson, Mitsuo Takeda

Wei Wang, Tim K. Lee, Steen G. Hanson, Mitsuo Takeda, "Generation and development of polarization speckle based on random walks of polarization phasor," Proc. SPIE 11052, Third International Conference on Photonics and Optical Engineering, 110521N (24 January 2019); doi: 10.1117/12.2523353

SPIE Event: The International Conference on Photonics and Optical Engineering, 2018, Xi'an, China 


\title{
Generation and Development of Polarization Speckle Based on Random Walks of Polarization Phasor
}

\author{
Wei Wang, ${ }^{\mathrm{a}, \mathrm{b}, *}$ Tim K. Lee, ${ }^{\mathrm{c}, \mathrm{d}}$ Steen G. Hanson, ${ }^{\mathrm{e}}$ and Mitsuo Takeda ${ }^{\mathrm{b}, \mathrm{f}}$ \\ ${ }^{a}$ Institute of Photonics and Quantum Sciences, School of Engineering and Physical Science, \\ Heriot-Watt University, Edinburgh, United Kingdom, EH14 4AS; \\ b international Center for Optical Research and Education (iCORE), Xi'an Technological University, \\ $\mathrm{Xi}$ 'an 710032, China \\ ${ }^{\mathrm{c}}$ Cancer Control Research Program, BC Cancer Agency, Vancouver, BC V5Z 1L3, Canada \\ ${ }^{\mathrm{d}}$ Department of Dermatology and Skin Science, University of British Columbia, Vancouver, \\ BC V5Z4E8, Canada \\ e DTU Fotonik, Department of Photonics Engineering, \\ Technical University of Denmark, Dk-4000 Roskilde, Denmark \\ ${ }^{\mathrm{f}}$ Center for Optical Research and Education (CORE), Utsunomiya University, 7-1-2 Yoto, \\ Utsunomiya, Tochigi 321-8585, Japan
}

\begin{abstract}
In this paper, we review our recent work on the statistical properties of polarization speckle with its unique properties of random polarization states fluctuating in space. From random walks of polarization phasor, we provide the origins of the polarization speckle by taking the vector nature into account for stochastic electric fields. Based on the random polarization phasor sum as an extension of conventional random phasor sum, the first and second moments of the Stokes parameters of the resultant polarization speckle have been examined. Two types of devices for polarization optics, i.e. rough-surfaced retardation plate and random polarizer array, will be introduced as the physical realization of the desired phenomena of polarization speckles. Some statistical properties of the stochastic electric fields are reviewed in order to understand the performance of these two polarization optics devices for generation and development of the polarization speckle.
\end{abstract}

Keywords: Speckles, polarization, random walk, mutual coherence tensor, statistical optics

\section{INTRODUCTION}

Extensive studies have been made on the basic properties and applications of laser speckle since continuous-wave lasers became commercially available in the early 1960s [1-3]. The term speckle pattern is usually associated with the fine-scale granular distribution of a light intensity pattern arising from the interference of coherently superposed multiple random optical fields. In the majority of studies on speckle phenomena, these random optical fields have been treated as scalar optical fields with their main interest being in the statistical properties and applications of the intensity distribution of the speckle patterns. Recently, statistical properties of random electric vector fields have come to attract new interest because of their theoretical importance and practical applications. Statistical phenomena of random electric vector fields referred to as Polarization Speckle have relevance to the theories of speckles, polarization and coherence $[3,4]$. In this paper, we review our recent work on the generation and development of polarization speckle from random walks of polarization phasor by taking the vector nature into account for stochastic electric fields. Two types of devices for polarization optics, i.e. rough-surfaced retardation plate and random polarizer array, are reviewed as the physical realization of the desired phenomena and some corresponding statistical properties of the stochastic electric fields are introduced to understand their performance for generation and development of polarization speckle.

*w.wang@hw.ac.uk Tel: +44 (0) 131451 3141; Fax: +44 (0) 1314513129

Third International Conference on Photonics and Optical Engineering, edited by Ailing Tian, Proc. of SPIE Vol. 11052, 110521N · C 2019 SPIE · CCC code: 0277-786X/19/\$18 · doi: 10.1117/12.2523353 


\section{POLARIZATION SPECKLE AND ITS ORIGINS}

Similar to conventional laser speckle composed of a multitude of independently phased additive complex components, polarization speckle known as stochastic electric fields is a vectorial signal, which could be understood as a sum of many contributions from polarization phasors with random vector directions (random polarization states) and random complex amplitudes (random amplitudes and random phases) representing a monochromatic or nearly monochromatic vectorial electric field disturbance. Figure 1 (a) and (b) give physical origin of polarization speckle generated from two types of polarization optics elements, i.e. rough-surfaced wave plate and random polarizer array. When nearly monochromatic light is passing through such devices, the electric field resulting at any moderately distant point consists of many coherent components of polarization wavelets, each arising from a different microscopic element of the polarization optics element with different initial polarization state. With reference to Fig. 1, the distances travelled by these various polarization wavelets may differ by several or many wavelengths. Polarization interference of these coherent independent polarization wavelets results in the polarization speckle with granular intensity pattern and random polarization state in space.
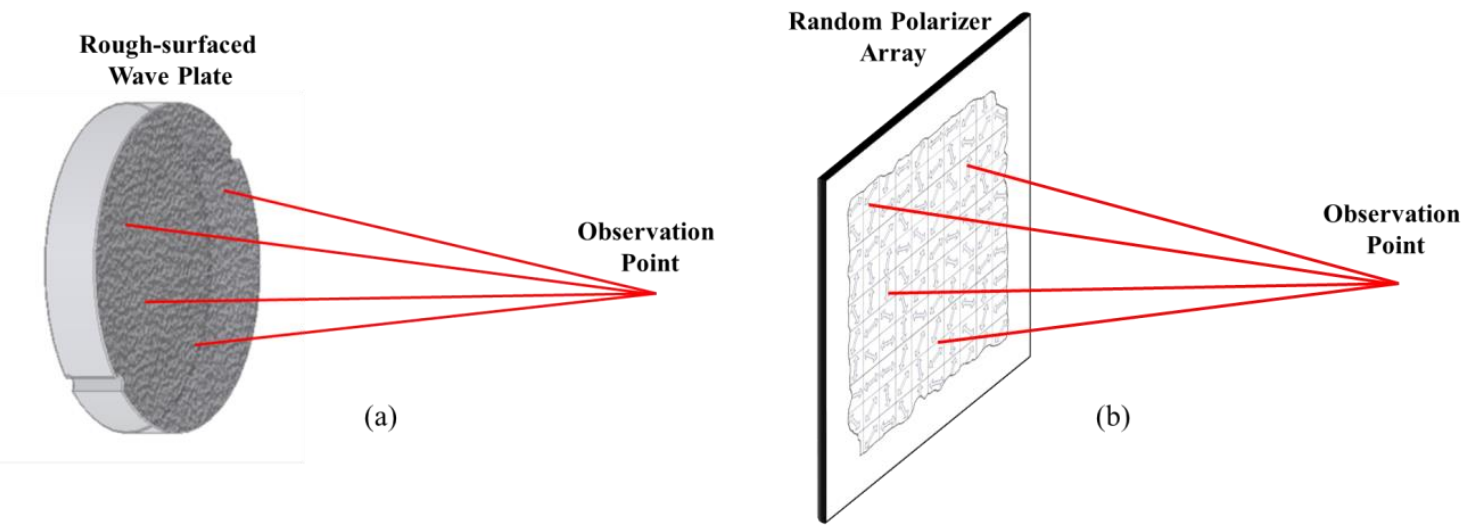

Figure 1. Physical generation of polarization speckle from (a) rough-surfaced wave plate, (b) random polarizer array

\section{DOUBLE RANDOM WALKS IN COMPLEX PLANES}

In this session, we present some of the details of the derivation that leads to the expressions for the mean and the moments of the Stokes parameters for polarization speckle based on random walks of the polarization phasor. Figure 2 illustrates an example of polarization phasor sum based on the vector random walks for $\tilde{E}_{x}$ and $\tilde{E}_{y}$, respectively. When these components of the polarization phasors are added vectorially together, they constitute what is known as a "vectorial random walk." The sums for each polarization component may be large or small, depending on the relative phases of the various components of the sum, and in particular whether constructive or destructive interference dominates the sum, and therefore the polarization state will be determined from the resultants of sums for these two polarization components of the vectorial electric field. Given that the complex electric field of the random walk can be expressed as [5]

$$
\mathbf{E}=\tilde{E}_{x} \hat{x}+\tilde{E}_{y} \hat{y}=a_{x} e^{i \phi_{x}} \hat{x}+a_{y} e^{i \phi_{y}} \hat{y}=\frac{1}{\sqrt{N}} \sum_{n=1}^{N} \mathbf{E}_{n}=\frac{1}{\sqrt{N}} \sum_{n=1}^{N}\left(a_{x n} e^{i \phi_{x i n}} \hat{x}+a_{y n} e^{i \phi_{y n}} \hat{y}\right),
$$

where $\mathbf{E}$ represents the resultant polarization phasor (a complex electric field vector), $\tilde{E}_{x}$ and $\tilde{E}_{y}$ are two Cartesian components of $\mathbf{E}$ along the $\hat{x}$ and $\hat{y}$ directions, with them being unit vectors. $a_{x}$ and $a_{y}$ are the lengths and $\phi_{x}$ and $\phi_{y}$ are the phases of the resultant polarization components, respectively. $N$ represents the number of polarization phasor components in the random walk, $\mathbf{E}_{n}$ represents the $n$-th component of the polarization phasor in the sum (a complex vector), $\quad a_{k n}$ and $\phi_{k n}$ are the length and phase for $k=x$ or $y$ components of $\vec{E}_{n}$, respectively. The scaling factor $1 / \sqrt{N}$ is introduced here in order to preserve finite energy, i.e. $\left\langle\left.\mathbf{E}\right|^{2}>\right.$, even when the number of component polarization phasors approaches infinity. 

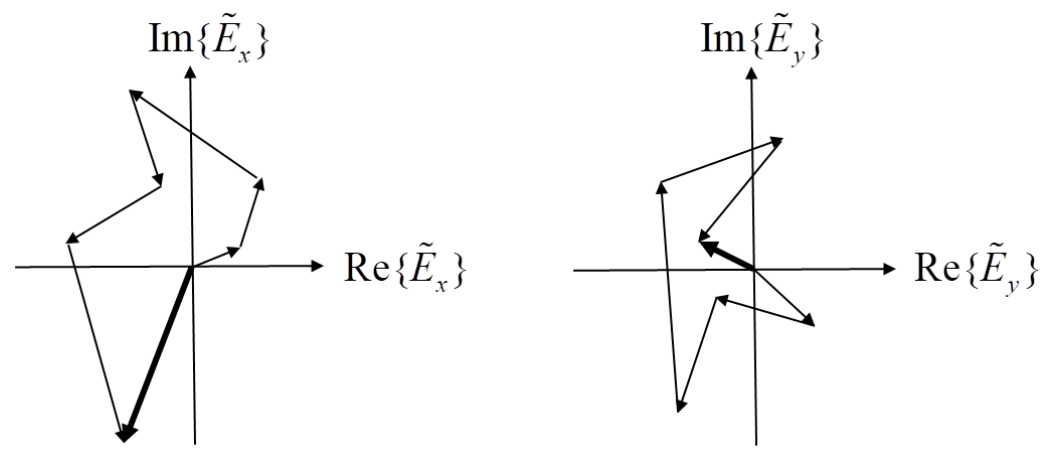

Figure 2 Schematic diagram of a random polarization phasor sum

Because of the great importance of the Stokes parameters in polarization optics, we present the Stokes parameters from their definitions and start our random polarization phasor sums as follows.

$$
\begin{aligned}
& S_{0}=\tilde{E}_{x} \tilde{E}_{x}^{*}+\tilde{E}_{y} \tilde{E}_{y}^{*}=\frac{1}{N} \sum_{n=1}^{N} \sum_{m=1}^{N}\left[a_{x n} a_{x m} e^{i\left(\phi_{x n}-\phi_{x m}\right)}+a_{y n} a_{y m} e^{i\left(\phi_{y n}-\phi_{y m}\right)}\right] \\
& S_{1}=\tilde{E}_{x} \tilde{E}_{x}^{*}-E_{y} \tilde{E}_{y}^{*}=\frac{1}{N} \sum_{n=1}^{N} \sum_{m=1}^{N}\left[a_{x n} a_{x m} e^{i\left(\phi_{x n}-\phi_{x m}\right)}-a_{y n} a_{y m} e^{i\left(\phi_{y n}-\phi_{y m}\right)}\right] \\
& S_{2}=\tilde{E}_{x}^{*} \tilde{E}_{y}+\tilde{E}_{y}^{*} \tilde{E}_{x}=\frac{1}{N} \sum_{n=1}^{N} \sum_{m=1}^{N}\left[a_{y n} a_{x m} e^{i\left(\phi_{y n}-\phi_{x m}\right)}+a_{x n} a_{y m} e^{i\left(\phi_{x n}-\phi_{y m}\right)}\right] \\
& S_{3}=i\left(\tilde{E}_{x}^{*} \tilde{E}_{y}-\tilde{E}_{y}^{*} \tilde{E}_{x}\right)=\frac{i}{N} \sum_{n=1}^{N} \sum_{m=1}^{N}\left[a_{y n} a_{x m} e^{i\left(\phi_{y n}-\phi_{x m}\right)}-a_{x n} a_{y m} e^{i\left(\phi_{x n}-\phi_{y m}\right)}\right]
\end{aligned}
$$

where $*$ indicates a complex conjugate. Allowing for the moment arbitrary statistics for both the amplitudes $a_{x n}$ and $a_{y n}$ and the phases $\phi_{x n}$ and $\phi_{y n}$, but assuming that amplitude and phase of the $n^{\text {th }}$ elementary polarization phasor are statistically independent of each other and of the amplitudes and phases of all other elementary polarization phasor. We can express the mean stokes parameters as

$$
\begin{aligned}
& <S_{0}>=\frac{1}{N} \sum_{n=1}^{N} \sum_{m=1}^{N}\left[<a_{x n} a_{x m} e^{i\left(\phi_{x n}-\phi_{x m}\right)}>+<a_{y n} a_{y m} e^{i\left(\phi_{y n}-\phi_{y m}\right)}>\right]=<a_{x}^{2}>+<a_{y}^{2}>+(N-1)<a_{x}>^{2} M_{x}^{\phi}(-1) M_{x}^{\phi}(1) \\
& +(N-1)<a_{y}>^{2} M_{y}^{\phi}(-1) M_{y}^{\phi}(1), \\
& <S_{1}>=\frac{1}{N} \sum_{n=1}^{N} \sum_{m=1}^{N}\left[<a_{x n} a_{x m} e^{i\left(\phi_{x n}-\phi_{x m}\right)}>-<a_{y n} a_{y m} e^{i\left(\phi_{y n}-\phi_{y m}\right)}>\right]=<a_{x}^{2}>-<a_{y}^{2}>+(N-1)<a_{x}>^{2} M_{x}^{\phi}(-1) M_{x}^{\phi}(1) \\
& -(N-1)<a_{y}>^{2} M_{y}^{\phi}(-1) M_{y}^{\phi}(1), \\
& <S_{2}>=\frac{1}{N} \sum_{n=1}^{N} \sum_{m=1}^{N}\left[<a_{x n} a_{y m} e^{i\left(\phi_{x n}-\phi_{y m}\right)}>+<a_{y n} a_{x m} e^{i\left(\phi_{y n}-\phi_{x m}\right)}>\right]=N<a_{x}><a_{y}>\left[M_{x}^{\phi}(1) M_{y}^{\phi}(-1)+M_{x}^{\phi}(-1) M_{y}^{\phi}(1)\right] \\
& <S_{3}>=\frac{i}{N} \sum_{n=1}^{N} \sum_{m=1}^{N}\left[<a_{y n} a_{x m} e^{i\left(\phi_{y n}-\phi_{x m}\right)}>-<a_{x n} a_{y m} e^{i\left(\phi_{x n}-\phi_{y m}\right)}>\right]=-i N<a_{x}><a_{y}>\left[M_{x}^{\phi}(1) M_{y}^{\phi}(-1)-M_{x}^{\phi}(-1) M_{y}^{\phi}(1)\right] .
\end{aligned}
$$

When Eqs. (3.1) (3.4) have been derived, we have assumed that all $a_{k n}$ ( $k=x$ or $y$ ) are identically distributed, with mean $<a_{k}>$ and second moment $\left\langle a_{k}^{2}\right\rangle$. We have also assumed that all $\phi_{k n}$ are identically distributed and therefore have common characteristic function $M_{k}^{\phi}(\omega)$ given by $M_{k}^{\phi}(\omega)=<\exp \left(j \omega \phi_{k}\right)>$. We now turn our attention to the more difficult problem of calculating the second moment of the Stokes parameters $<S_{i}^{2}>(i=0 \sim 3)$. 


$$
\begin{aligned}
& \overline{S_{0}^{2}}=\frac{1}{N^{2}} \sum_{n=1}^{N} \sum_{m=1}^{N} \sum_{p=1}^{N} \sum_{q=1}^{N} \overline{\left[a_{x n} a_{x m} e^{i\left(\phi_{x n}-\phi_{x m}\right)}+a_{y n} a_{y m} e^{i\left(\phi_{y m}-\phi_{y m}\right)}\right] \times\left[a_{x p} a_{x q} e^{i\left(\phi_{x p}-\phi_{x q}\right)}+a_{y p} a_{y q} e^{i\left(\phi_{y p}-\phi_{y q}\right)}\right]}, \\
& \overline{S_{1}^{2}}=\frac{1}{N^{2}} \sum_{n=1}^{N} \sum_{m=1}^{N} \sum_{p=1}^{N} \sum_{q=1}^{N} \overline{\left[a_{x n} a_{x m} e^{i\left(\phi_{x n}-\phi_{x m}\right)}-a_{y n} a_{y m} e^{i\left(\phi_{y m}-\phi_{y m}\right)}\right] \times\left[a_{x p} a_{x q} e^{i\left(\phi_{x p}-\phi_{x q}\right)}-a_{y p} a_{y q} e^{i\left(\phi_{y p}-\phi_{y g}\right)}\right]}, \\
& \overline{S_{2}^{2}}=\frac{1}{N^{2}} \sum_{n=1}^{N} \sum_{m=1}^{N} \sum_{p=1}^{N} \sum_{q=1}^{N} \overline{\left[a_{y n} a_{x m} e^{i\left(\phi_{y n}-\phi_{x m}\right)}+a_{x n} a_{y m} e^{i\left(\phi_{x n}-\phi_{y m}\right)}\right] \times\left[a_{y p} a_{x q} e^{i\left(\phi_{y p}-\phi_{x q}\right)}+a_{x p} a_{y q} e^{i\left(\phi_{x p}-\phi_{y q}\right)}\right]}, \\
& \overline{S_{3}^{2}}=\frac{1}{N^{2}} \sum_{n=1}^{N} \sum_{m=1}^{N} \sum_{p=1}^{N} \sum_{q=1}^{N} \overline{\left[a_{y n} a_{x m} e^{i\left(\phi_{y n}-\phi_{x m}\right)}-a_{x n} a_{y m} e^{i\left(\phi_{x m}-\phi_{y m}\right)}\right] \times\left[a_{y p} a_{x q} e^{i\left(\phi_{y p}-\phi_{x q}\right)}-a_{x p} a_{y q} e^{i\left(\phi_{x p}-\phi_{y q}\right)}\right]},
\end{aligned}
$$

where we have assumed again that the amplitudes and phases of the component polarization phasors are statistically independent. For these summations corresponding to each stokes parameter, there are 15 different cases that must be considered, as follows $[2,3]$.
(1) $n=m=p=q$
$N$ terms
(2) $n=m, p=q, n \neq p \quad N(N-1)(N-2)$ terms
(3) $n=m, p \neq q \neq n \quad N(N-1)(N-2)$ terms
(4) $n=p, m=q, n \neq m \quad N(N-1)$ terms
(5) $n=p, m \neq q \neq n \quad N(N-1)(N-2)$ terms
(6) $n=q, m=p, n \neq m \quad N(N-1)$ terms
(7) $n=q, m \neq p \neq n \quad N(N-1)(N-2)$ terms
(8) $n=m=p, n \neq q \quad N(N-1)$ terms
(9) $n=m=q, n \neq p \quad N(N-1)$ terms
(10) $n=p=q, n \neq m \quad N(N-1)$ terms
(11) $p=q=m, n \neq m \quad N(N-1)$ terms
(12) $n \neq m \neq p \neq q \quad N(N-1)(N-2)(N-3)$ terms
(13) $p=q, n \neq m \neq p \quad N(N-1)(N-2)$ terms
(14) $m=q, n \neq m \neq p \quad N(N-1)(N-2)$ terms
(15) $m=p, n \neq m \neq q \quad N(N-1)(N-2)$ terms

After summation of all 15 terms, we have our desired second moments for each individual Stokes parameter. They are

$$
\begin{aligned}
<S_{0}^{2}> & =\frac{1}{N}\left\{<a_{x}^{4}>+<a_{y}^{4}>+2(N-1)\left[<a_{x}^{2}>^{2}+<a_{y}^{2}>^{2}\right]+2 N<a_{x}^{2}><a_{y}^{2}>+\left(N^{3}-6 N^{2}+11 N-6\right)\left[<a_{x}>^{4}\right.\right. \\
& \left.\times\left(M_{x}^{\phi}(1)\right)^{4}+<a_{y}>^{4}\left(M_{y}^{\phi}(1)\right)^{4}\right]+\left(M_{x}^{\phi}(1)\right)^{2}\left[4(N-1)<a_{x}><a_{x}^{3}>+2\left(N^{2}-3 N+2\right)<a_{x}>^{2}<a_{x}^{2}>\right. \\
& \left.\times\left(M_{x}^{\phi}(2)+2\right)+2 N(N-1)<a_{x}>^{2}<a_{y}^{2}>\right]+\left(M_{y}^{\phi}(1)\right)^{2}\left[4(N-1)<a_{y}><a_{y}^{3}>+2\left(N^{2}-3 N+2\right)\right. \\
& \left.\times<a_{y}>^{2}<a_{y}^{2}>\left(M_{y}^{\phi}(2)+2\right)+2 N(N-1)<a_{y}>^{2}<a_{x}^{2}>\right]+(N-1)\left[<a_{x}^{2}>^{2}\left(M_{x}^{\phi}(2)\right)^{2}+<a_{y}^{2}>^{2}\left(M_{y}^{\phi}(2)\right)^{2}\right] \\
& \left.+2 N(N-1)^{2}<a_{x}>^{2}<a_{y}>^{2} \times\left(M_{x}^{\phi}(1)\right)^{2}\left(M_{y}^{\phi}(1)\right)^{2}\right\}, \\
<S_{1}^{2}> & =\frac{1}{N}\left\{<a_{x}^{4}>+<a_{y}^{4}>+2(N-1)\left[<a_{x}^{2}>^{2}+<a_{y}^{2}>^{2}\right]-2 N<a_{x}^{2}><a_{y}^{2}>+\left(N^{3}-6 N^{2}+11 N-6\right)\left[<a_{x}>^{4}\right.\right. \\
& \left.\times\left(M_{x}^{\phi}(1)\right)^{4}+<a_{y}>^{4}\left(M_{y}^{\phi}(1)\right)^{4}\right]+\left(M_{x}^{\phi}(1)\right)^{2}\left[4(N-1)<a_{x}><a_{x}^{3}>+2\left(N^{2}-3 N+2\right)<a_{x}>^{2}<a_{x}^{2}>\right. \\
& \left.\times\left(M_{x}^{\phi}(2)+2\right)-2 N(N-1)<a_{x}>^{2}<a_{y}^{2}>\right]+\left(M_{y}^{\phi}(1)\right)^{2}\left[4(N-1)<a_{y}><a_{y}^{3}>+2\left(N^{2}-3 N+2\right)\right. \\
& \left.\times<a_{y}>^{2}<a_{y}^{2}>\left(M_{y}^{\phi}(2)+2\right)-2 N(N-1)<a_{y}>^{2}<a_{x}^{2}>\right]+(N-1)\left[<a_{x}^{2}>^{2}\left(M_{x}^{\phi}(2)\right)^{2}+<a_{y}^{2}>^{2}\left(M_{y}^{\phi}(2)\right)^{2}\right] \\
& \left.-2 N(N-1)^{2}<a_{x}>^{2}<a_{y}>^{2}\left(M_{x}^{\phi}(1)\right)^{2}\left(M_{y}^{\phi}(1)\right)^{2}\right\},
\end{aligned}
$$




$$
\begin{aligned}
<S_{2}^{2}>= & 2(N-1)<a_{x}>^{2}\left(M_{x}^{\phi}(1)\right)^{2}\left[2(N-1)<a_{y}>^{2}\left(M_{y}^{\phi}(1)\right)^{2}+<a_{y}^{2}>\left(1+M_{y}^{\phi}(2)\right)\right]+<a_{x}^{2}>\left[2(N-1)<a_{y}>^{2}\right. \\
& \left.\times\left(1+M_{x}^{\phi}(2)\right)\left(M_{y}^{\phi}(1)\right)^{2}+2<a_{y}^{2}>\left(1+M_{x}^{\phi}(2) M_{y}^{\phi}(2)\right)\right], \\
<S_{3}^{2}>= & -2(N-1)<a_{x}>^{2}<a_{y}^{2}>\left(M_{x}^{\phi}(1)\right)^{2}\left(M_{y}^{\phi}(2)-1\right)-<a_{x}^{2}>\left[2(N-1)<a_{y}>^{2}\left(M_{x}^{\phi}(2)-1\right)\left(M_{y}^{\phi}(1)\right)^{2}\right. \\
& \left.+2<a_{y}^{2}>\left(M_{x}^{\phi}(2) M_{y}^{\phi}(2)-1\right)\right] .
\end{aligned}
$$

When these results have been derived, we make use of the Hermitian symmetry, $M_{k}^{\phi}(-\omega)=\left[M_{k}^{\phi}(\omega)\right]^{*}$ due to the fact that the probability density functions of $\varphi_{k}$ is real-valued. All these resulting expressions in Eqs. (5.1)-(5.4) are sufficient to understand the mean and the moments of the Stokes parameters for polarization speckle if some assumptions for $\varphi_{k}$ are made.

\section{GENERATION AND DEVELOPMENT OF POLARZATION SPECKLES WITH A ROUGH-SURFACED WAVE PLATE}

In this section, we will review our recent work on the generation and development of polarization speckle from a roughsurfaced wave plate, and explore how statistical properties polarization speckle depend on the microstructure of the rough surface of birefringent materials [6,7].

As shown in Fig. 3, we assume free-space transmission geometry for surface polarization scattering, where an example of a cross-section of the birefringent material with a typical random surface height fluctuation has been given. Just as the laser speckle generated from the rough surface, the relationship between these height variations of the rough-surfaced retardation plate and the associated amplitude variations of the scattered electric field is in general extremely complicated. It is influenced by variations of surface slope, shadowing of retardation plate, multiple scattering and reflection inside the birefringent material, and polarization effects introduced to the scattered wave travelling in the retardation plate in arbitrary directions with reference to the orientation of fast/slow axis of the birefringent material. For analysis purpose, in this letter we will adopt an oversimplified model to give some physical insight into the relationship between the surface height fluctuations for the rough-surfaced retardation plate and the polarization fluctuations of the scattered electric field.

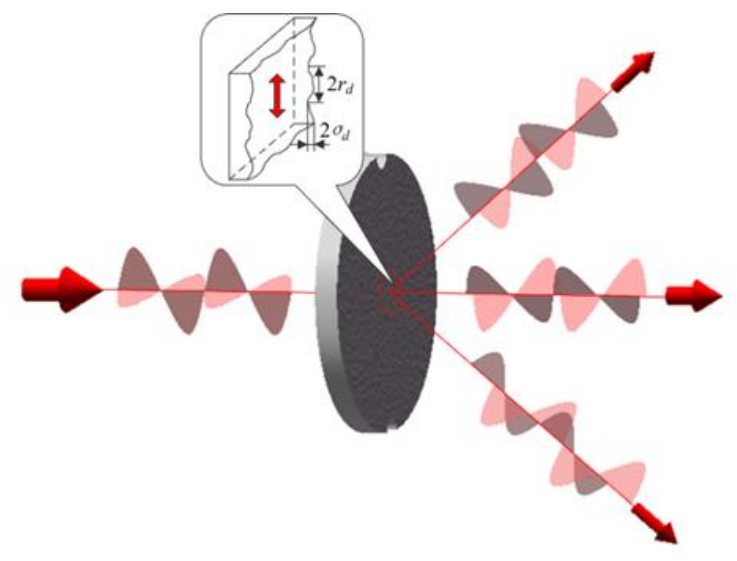

Figure 3 Schematic diagram of polarization speckle from a rough surfaced wave plate

Let the scattered electric field immediately behind the rough surface, $\mathbf{E}^{t}(\mathbf{r})$, be related to the incident electric vector $\mathbf{E}^{i}(\mathbf{r})$ through the Jones matrix $\mathbf{T}(\mathbf{r})$ for a birefringent material with its fast/slow axis aligned along the $\hat{y}$ direction given by

$$
\mathbf{T}(\mathbf{r})=\left(\begin{array}{cc}
e^{-j \phi_{x}(\mathbf{r})} & 0 \\
0 & e^{-j \phi_{y}(\mathbf{r})}
\end{array}\right),
$$

where $\phi_{m}(m=x, y)$ is the effective phase delay for the $\hat{x}$ or $\hat{y}$ components of the electric field introduced by an optical path passing through the retardation plate with surface height fluctuation and the remaining region being free space. Under 
the assumption that the coherence property of the incident illumination field and the correlation property of the depolarizer are statistically independent, we have the transmission coherence matrix $\boldsymbol{W}^{t}\left(\mathbf{r}_{1}, \mathbf{r}_{2}\right)$ of the modulated fields just behind the depolarizer. That is

$$
W^{t}\left(\mathbf{r}_{1}, \mathbf{r}_{2}\right)=\left(\begin{array}{ll}
W_{x x}^{i}\left(\mathbf{r}_{1}, \mathbf{r}_{2}\right)<e^{j \Delta \phi_{x x}\left(\mathbf{r}_{1}, \mathbf{r}_{2}\right)}>W_{x y}^{i}\left(\mathbf{r}_{1}, \mathbf{r}_{2}\right)<e^{j \Delta \phi_{y y}\left(\mathbf{r}_{1}, \mathbf{r}_{2}\right)}> \\
W_{y x}^{i}\left(\mathbf{r}_{1}, \mathbf{r}_{2}\right)<e^{j \Delta \phi_{y x}\left(\mathbf{r}_{1}, \mathbf{r}_{2}\right)}>W_{y y}^{i}\left(\mathbf{r}_{1}, \mathbf{r}_{2}\right)<e^{j \Delta \phi_{y y}\left(\mathbf{r}_{1}, \mathbf{r}_{2}\right)}>
\end{array}\right),
$$

with the angular brackets $\langle\cdots\rangle$ above denoting "ensemble average". For demonstration purposes only, and without loss of generality, we assume that the incident light is a linearly polarized, spatially coherent, plane wave for which the coherence matrix is given by

$$
\boldsymbol{W}^{i}\left(\mathbf{r}_{1}, \mathbf{r}_{2}\right)=I_{0}\left(\begin{array}{cc}
\cos ^{2} \theta & \cos \theta \sin \theta \\
\cos \theta \sin \theta & \sin ^{2} \theta
\end{array}\right)
$$

where $I_{0}$ is the on-axis intensity of the incident field and $\theta$ is the linear polarization angle with the $\hat{x}$-axis. Some further progress can be made for Eq. (3) when certain assumptions for the effective phase delay and the correlation function of the surface thickness are specified [2-3]. For simplicity, the assumption is usually made that the effective phase delays (or equivalently the surface height fluctuations) is a Gaussian random process. We have

$$
\begin{aligned}
<e^{j \Delta \phi_{i m}}>= & \left\langle\exp \left\{j(2 \pi / \lambda)\left[\left(n_{l}-1\right)\left(\bar{d}+d\left(\mathbf{r}_{1}\right)\right)-\left(n_{m}-1\right)\left(\bar{d}+d\left(\mathbf{r}_{1}\right)\right)\right]\right\}\right\rangle \\
= & \exp \left\{(j 2 \pi / \lambda)\left(n_{l}-n_{m}\right) \bar{d}\right\} \exp \left\{-\left(2 \pi^{2} / \lambda^{2}\right)\left[\left(n_{l}-1\right)^{2}<d^{2}\left(\mathbf{r}_{1}\right)>\right.\right. \\
& \left.\left.+\left(n_{m}-1\right)^{2}<d^{2}\left(\mathbf{r}_{2}\right)>-2\left(n_{l}-1\right)\left(n_{m}-1\right)<d\left(\mathbf{r}_{1}\right) d\left(\mathbf{r}_{2}\right)>\right]\right\},
\end{aligned}
$$

where $\lambda$ is the wavelength in vacuum, $d(r)$ is the zero mean Gaussian random thickness variation around the average thickness $\bar{d}$ of the birefringent plate, $n_{l}$ and $n_{m}$ are the refractive indices for the birefringent material. Another assumption is that the correlation function of the surface thickness also takes Gaussian form.

$$
<d\left(\mathbf{r}_{1}\right) d\left(\mathbf{r}_{2}\right)>=\sigma_{d}^{2} \exp \left\{-\left|\mathbf{r}_{1}-\mathbf{r}_{2}\right|^{2} / r_{d}^{2}\right\},
$$

where $\sigma_{d}^{2}$ and $r_{d}$ are mutually independent quantities indicating the variance of $d(r)$ and the radius at which the normalized surface thickness correlation falls to $1 / e$, respectively. Therefore, the transmission coherence tensor of the scattered electric field immediately behind the scattering layer becomes a complex Hermitian matrix.

$$
\begin{aligned}
W_{x x}^{t}(\Delta r)= & I_{0} \cos ^{2} \theta \exp \left\{-4 \pi^{2}\left(\sigma_{d} / \lambda\right)^{2}\left(n_{x}-1\right)^{2}\left[1-\exp \left(-\Delta r^{2} / r_{d}^{2}\right)\right]\right\}, \\
W_{y y}^{t}(\Delta r)= & I_{0} \sin ^{2} \theta \exp \left\{-4 \pi^{2}\left(\sigma_{d} / \lambda\right)^{2}\left(n_{y}-1\right)^{2}\left[1-\exp \left(-\Delta r^{2} / r_{d}^{2}\right)\right]\right\}, \\
W_{x y}^{t}(\Delta r)= & {\left[W_{y x}^{t}(\Delta r)\right]^{*}=I_{0} \sin \theta \cos \theta \exp \left\{j 2 \pi \bar{d}\left(n_{x}-n_{y}\right) / \lambda\right\} \exp \left\{-2 \pi^{2}\left(\sigma_{d} / \lambda\right)^{2}\right.} \\
& {\left.\left[\left(n_{x}-1\right)^{2}+\left(n_{y}-1\right)^{2}-2\left(n_{x}-1\right)\left(n_{y}-1\right) \exp \left(-\Delta r^{2} / r_{d}^{2}\right)\right]\right\} . }
\end{aligned}
$$

When Equations (11.1)-(11.3) have been derived, the surface of the polarization scattering spot is assumed to be rough and its statistics being wide-sense stationary, where its correlation function depends only on the differences of measurement coordinates: $\Delta r=\left|\mathbf{r}_{1}-\mathbf{r}_{2}\right|$. These results provide us with a specific relationship between the correlation properties of the transmitted electric field and the micro-structure of the rough surface of the birefringent material. Under the assumption of a large surface roughness with large phase variance owing to the smaller lateral distance required to obtain a phase difference of $2 \pi$, we can make further progress. In this case the second exponential terms in Eq. (7) may be series expanded, and only the first two terms in the series are essential. For $\left(n_{l}-1\right)\left(n_{m}-1\right) k^{2} \sigma_{d}{ }^{2}>(2 \pi)^{2},(l, m=x, y)$, we have 


$$
\left\langle\exp \left\{j \Delta \phi_{l m}\right\}\right\rangle=\exp \left\{j 2 \pi \bar{d}\left(n_{x}-n_{y}\right) / \lambda\right\} \exp \left\{-k^{2} \sigma_{d}^{2} / 2\left[\left(n_{l}-n_{m}\right)^{2}+2\left(n_{l}-1\right)\left(n_{m}-1\right)|\Delta \mathbf{r}|^{2} / r_{d}^{2}\right]\right\} .
$$

The approximation in Eq. (13) has resulted in breaking down the double exponential into a single exponential facilitating further mathematical analysis in order later to arrive at an analytical solutions for the propagation of the coherence matrix. Under the paraxial approximation, the propagation of the coherence matrix through a complex-valued ABCD optical system is given by

$$
W^{o}\left(\mathbf{p}_{1}, \mathbf{p}_{2}\right)=\iint W^{t}\left(\mathbf{r}_{1}, \mathbf{r}_{2}\right) G^{*}\left(\mathbf{r}_{1}, \mathbf{p}_{1}\right) G\left(\mathbf{r}_{2}, \mathbf{p}_{2}\right) d \mathbf{r}_{1} d \mathbf{r}_{2} .
$$

where the superscript ${ }^{O}$ indicates the field in the observation plane, and the Green's function in the matrix formalism is

$$
G(\mathbf{r}, \mathbf{p})=-\frac{j k}{2 \pi B} \exp \left\{-\frac{j k}{2 B}\left(A|\mathbf{r}|^{2}-2 \mathbf{r} \cdot \mathbf{p}+D|\mathbf{p}|^{2}\right)\right\} .
$$

In the equation above, $j$ is the imaginary unit and the $A, B$ and $D$ are complex values that can be determined by multiplying the matrices for all the individual optical components in the optical train, i.e. lenses, free space propagation and apertures [8-9]. The benefit of the above expression in Eq. (13) is that it usually enables us to give an analytical result, covering a broad range of optical systems. In arriving at the above expression, we have tacitly assumed that the refractive indices in the input and output planes are identical, but not necessarily unity. As an example, we will consider a typical case of free space propagation over a distance $z$ with a preceding aperture of size $r_{s}$ to model the illuminating spot size, and thus the corresponding $\mathrm{ABCD}$ matrix is

$$
\left(\begin{array}{cc}
1-j z / z_{R} & z \\
-j / z_{R} & 1
\end{array}\right)
$$

where $z_{R}$ is the Rayleigh range $z_{R}=k r_{s}^{2} / 2$. By substituting these elements of the ABCD matrix into Eq. (14), one can study the changes in the degree of coherence and the degree of polarization for free space propagation. Based on the coherence matrix derived above, two parameters of primary interest, i.e. the degree of coherence $\eta$ and spatial degree of polarization $P_{s}$ could be estimated [11-12]. They are

$$
\eta\left(\mathbf{r}_{1}, \mathbf{r}_{2}\right)=\frac{\operatorname{tr} \boldsymbol{W}\left(\mathbf{r}_{1}, \mathbf{r}_{2}\right)}{\sqrt{\operatorname{tr} \boldsymbol{W}\left(\mathbf{r}_{1}, \mathbf{r}_{1}\right) \operatorname{tr} \boldsymbol{W}\left(\mathbf{r}_{2}, \mathbf{r}_{2}\right)}},
$$

and

$$
P(\mathbf{r})=\left\{1-\frac{4 \operatorname{det} \boldsymbol{W}(\mathbf{r}, \mathbf{r})}{[\operatorname{tr} \boldsymbol{W}(\mathbf{r}, \mathbf{r})]^{2}}\right\}^{1 / 2},
$$

where $t r$ and det indicate the trace and determinant of the matrix, respectively. Here the spatial average has been adopted for ensemble average when the coherence matrix in Eq. (7) and Eqs. (13) have been estimated. To provide physical insight into these results, we have presented some numerical examples by taking the following parameters: $\theta=\pi / 4, n_{x}=1.486$ and $n_{y}=1.658$ for the birefringent material: calcite [10].

Figure 4(a) gives the absolute values of the degree of coherence of the polarization speckle generated by the roughsurfaced retardation plate at two positions $\Delta \mathbf{p}=\mathbf{p}_{1}-\mathbf{p}_{2}$ located symmetrically with respect to the $\hat{z}$ axis along the normalized propagation distance. It can readily be seen that the degree of coherence takes a large value close to unity for two points located near the optical axis. Fig. 4(b) shows the degree of polarization of the polarization speckle for freespace propagation, plotted against the normalized propagation distance $z / z_{R}$, and the normalized lateral distance measured in spot size $p / r_{s}$. As expected, the degree of polarization changes appreciably depending both on the propagation distance $z$ and on the polarization angle of the incident beam. Instead of a uniform distribution for the modulated electric fields just behind the depolarizer, the spatial degree of polarization for the polarization speckle after propagation does not remain uniform. 


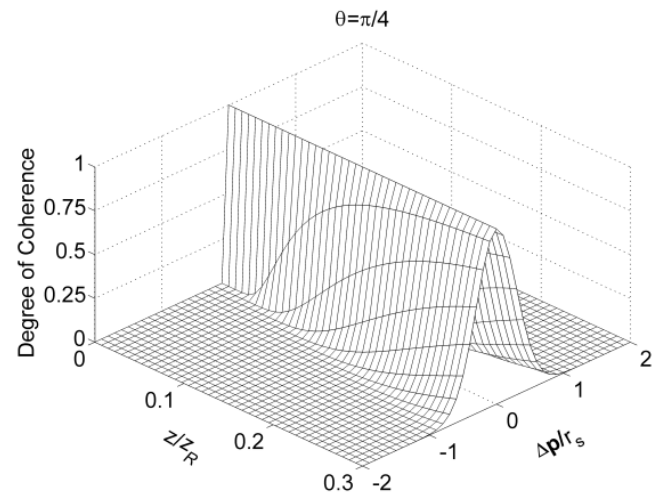

(a)

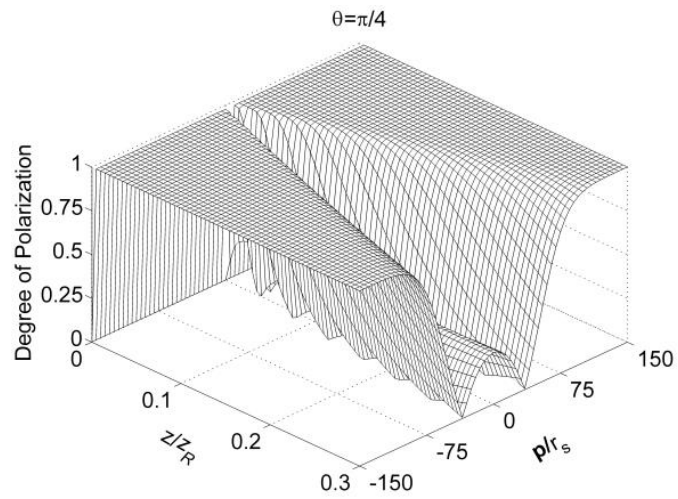

(b)

Figure 4 Degree of Coherence (a) and spatial degree polarization (b) for polarization speckle generated by a rough-surfaced wave plate

\section{GENERATION OF POLARIZATION SPECKLE WITH A RANDOMLY ORIENTED POLARIZER ARRAY}

In the last session, we have discussed the performance of the rough-surfaced wave plate for generation of polarization speckle. In this section, we will review our recent work on another type of depolarizer, namely a randomly oriented polarizer array, and explore how various statistical properties of polarization speckle depend on the distribution for random orientation angle of the polarizers [13]. As a specific example of this type of depolarizer, we consider the random polarizer array illustrated in Fig. 5. This structure consists of a multitude of contiguous $l \times l$ square cells. In each cell, the polarization orientation indicated by the small arrow has been randomly and independently chosen so that only the incident vector wave component parallel to the direction of the local arrow can pass. The random polarizer array itself may be regarded as being infinite in extent, although only a finite portion lies within the pupil of the optical system. Because of a lack of knowledge of the exact location of the depolarizer on the scale of a single cell, the assumption has been made that the statistical properties of the structure is wide-sense stationary over space. The location of the depolarizer with respect to the optical axis is chosen to be random with a uniform distribution of probability over any square cell.

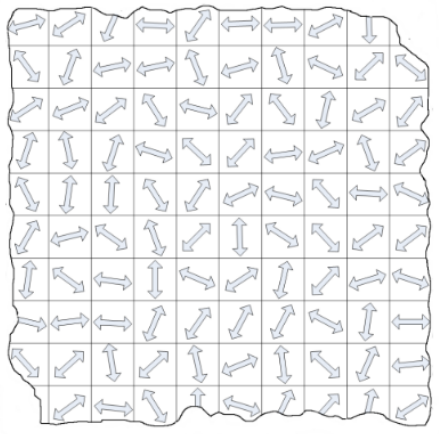

Figure 5. Diagram of depolarizer: random polarizer array

When the incident electric field passes through the depolarizer, the polarization state is modified accordingly, i.e., $\mathbf{E}^{t}(\mathbf{r})=\mathbf{T}(\mathbf{r}) \mathbf{E}^{i}(\mathbf{r})$ with the superscript $i$ or $t$ representing the incident or transmission field. With reference to the depolarizer pattern of Fig. 5, each square cell with a double-headed arrow inside indicates a polarizer with its axis of transmission at an angle $\varphi$ with the horizontal and the corresponding transmission matrix of such device is

$$
\mathbf{T}(\mathbf{r})=\left(\begin{array}{ll}
T_{x x}(\mathbf{r}) & T_{x y}(\mathbf{r}) \\
T_{y x}(\mathbf{r}) & T_{y y}(\mathbf{r})
\end{array}\right)=\left(\begin{array}{cc}
\cos ^{2} \varphi(\mathbf{r}) & \sin \varphi(\mathbf{r}) \cos \varphi(\mathbf{r}) \\
\cos \varphi(\mathbf{r}) \sin \varphi(\mathbf{r}) & \sin ^{2} \varphi(\mathbf{r})
\end{array}\right) .
$$


Let $\mathbf{W}^{i}\left(\mathbf{r}_{1}, \mathbf{r}_{2}\right)$ be the coherence matrix of the incident beam, then the coherence matrix $\mathbf{W}^{t}\left(\mathbf{r}_{1}, \mathbf{r}_{2}\right)$ of the modulated field just behind the depolarizer is given [14]

$$
\mathbf{W}^{t}\left(\mathbf{r}_{1}, \mathbf{r}_{2}\right)=\left\langle\mathbf{T}^{\dagger}\left(\mathbf{r}_{1}\right) \mathbf{W}^{i}\left(\mathbf{r}_{1}, \mathbf{r}_{2}\right) \mathbf{T}\left(\mathbf{r}_{2}\right)\right\rangle,
$$

where $\uparrow$ denotes the Hermitian conjugate. For any two points $\left(\mathbf{r}_{1}, \mathbf{r}_{2}\right)$ in a coherence matrix, there are two possible cases where they are in the same cell or in different cells of the random polarizer array, respectively. Please note that different cells have statistically independent polarization angle for each linear polarizer. Based on the concept of conditional probabilities, we can write the coherence matrix of the modulate fields as

$$
\mathbf{W}^{t}\left(\mathbf{r}_{1}, \mathbf{r}_{2}\right)={ }^{s} \mathbf{W}^{t}\left(\mathbf{r}_{1}, \mathbf{r}_{2}\right) \operatorname{Prob}\left\{\begin{array}{l}
\mathbf{r}_{1} \text { and } \mathbf{r}_{2} \text { are } \\
\text { in the same cell }
\end{array}\right\}+{ }^{D} \mathbf{W}^{t}\left(\mathbf{r}_{1}, \mathbf{r}_{2}\right) \operatorname{Prob}\left\{\begin{array}{l}
\mathbf{r}_{1} \text { and } \mathbf{r}_{2} \text { are } \\
\text { in different cells }
\end{array}\right\} .
$$

In the expression above, ${ }^{S} \mathbf{W}^{t}$ and ${ }^{D} \mathbf{W}^{t}$ have been introduced to denote the coherence matrices for the transmitted fields with points $\mathbf{r}_{1}$ and $\mathbf{r}_{2}$ falling into the same and different cells, respectively. Similar to the random checkerboard absorbing screen $[2,15]$, we are able to write the desired probabilities because of the uniform distribution of the absolute location of the depolarizer. They are

$$
\begin{aligned}
& \operatorname{Prob}\left\{\mathbf{r}_{1}, \mathbf{r}_{2} \text { in the same cell }\right\}=\wedge\left(\Delta r_{x} / l\right) \wedge\left(\Delta r_{y} / l\right), \\
& \operatorname{Prob}\left\{\begin{array}{c}
\mathbf{r}_{1} \text { and } \mathbf{r}_{2} \text { are in } \\
\text { different cells }
\end{array}\right\}=1-\wedge\left(\Delta r_{x} / l\right) \wedge\left(\Delta r_{y} / l\right),
\end{aligned}
$$

where $\Delta r_{x}$ and $\Delta r_{y}$ are the two components of the vector $\Delta \mathbf{r}=\mathbf{r}_{1}-\mathbf{r}_{2}$ along $\hat{x}$ and $\hat{y}$ directions, and the unit triangle function $\wedge(x)=1-|x|$ for $|x| \leq 1$ and zero otherwise. Note that the axis of polarizer in each cell is assumed random and independent from cell to cell. We have

$$
{ }^{S} \mathbf{W}^{t}\left(\mathbf{r}_{1}, \mathbf{r}_{2}\right)=\left(\begin{array}{ll}
{ }^{S} W_{x x}^{t}\left(\mathbf{r}_{1}, \mathbf{r}_{2}\right) & { }^{S} W_{x y}^{t}\left(\mathbf{r}_{1}, \mathbf{r}_{2}\right) \\
{ }^{s} W_{y x}^{t}\left(\mathbf{r}_{1}, \mathbf{r}_{2}\right) & { }^{s} W_{y y}^{t}\left(\mathbf{r}_{1}, \mathbf{r}_{2}\right)
\end{array}\right),
$$

with ${ }^{S} W_{f g}^{t}\left(\mathbf{r}_{1}, \mathbf{r}_{2}\right)=\sum_{l, m=x, y}\left\langle T_{f l}\left(\mathbf{r}_{1}\right) T_{g m}\left(\mathbf{r}_{2}\right)\right\rangle W_{l m}^{i}\left(\mathbf{r}_{1}, \mathbf{r}_{2}\right)$ for $(f, g=x, y)$, and

$$
{ }^{D} \mathbf{W}^{t}\left(\mathbf{r}_{1}, \mathbf{r}_{2}\right)=\left(\begin{array}{cc}
{ }^{D} W_{x x}^{t}\left(\mathbf{r}_{1}, \mathbf{r}_{2}\right) & { }^{D} W_{x y}^{t}\left(\mathbf{r}_{1}, \mathbf{r}_{2}\right) \\
{ }^{D} W_{y x}^{t}\left(\mathbf{r}_{1}, \mathbf{r}_{2}\right) & { }^{D} W_{y y}^{t}\left(\mathbf{r}_{1}, \mathbf{r}_{2}\right)
\end{array}\right) .
$$

with ${ }^{D} W_{f g}^{t}\left(\mathbf{r}_{1}, \mathbf{r}_{2}\right)=\sum_{l, m=x, y}\left\langle T_{f l}\left(\mathbf{r}_{1}\right)\right\rangle\left\langle T_{g m}\left(\mathbf{r}_{2}\right)\right\rangle W_{l m}^{i}\left(\mathbf{r}_{1}, \mathbf{r}_{2}\right)$.

When Eqs. (22) and (23) have been derived, we have made use of the fact that the coherence property of the electric fields and correlation property of the random polarizer array are statistically independent and the ensemble average denoted by angular brackets has been taken over the depolarizer. The coherence matrix for the modulated field just behind the depolarizer can be assessed only if some specific assumptions are made regarding the statistics of the polarization angle $\varphi$ for the random polarizer array. A case of most interest is the depolarizer whose stochastic polarization angle has a uniform probability distribution function within $[0, \pi)$. Under the assumption of $p_{\varphi}(\varphi)=1 / \pi$, the first-order and secondorder moments of the elements of Jones Matrix in Eq. (18) have been evaluated and listed in the Table 1.

\begin{tabular}{|c|c|}
\hline$\left\langle T_{x x}\right\rangle=\left\langle\cos ^{2} \varphi(\mathbf{r})\right\rangle=1 / 2$ & $\left\langle T_{y y}\right\rangle=\left\langle\sin ^{2} \varphi(\mathbf{r})\right\rangle=1 / 2$ \\
\hline$\left\langle T_{x y}\right\rangle=\langle\cos \varphi(\mathbf{r}) \sin \varphi(\mathbf{r})\rangle=0$ & $\left\langle T_{y x}\right\rangle=\langle\cos \varphi(\mathbf{r}) \sin \varphi(\mathbf{r})\rangle=0$ \\
\hline$\left\langle T_{x x}^{2}\right\rangle=\left\langle\cos ^{4} \varphi(\mathbf{r})\right\rangle=3 / 8$ & $\left\langle T_{y y}^{2}\right\rangle=\left\langle\sin ^{4} \varphi(\mathbf{r})\right\rangle=3 / 8$ \\
\hline$\left\langle T_{x y}^{2}\right\rangle=\left\langle T_{y x}^{2}\right\rangle=\left\langle\sin ^{2} \varphi(\mathbf{r}) \cos ^{2} \varphi(\mathbf{r})\right\rangle=1 / 8$ & $\left\langle T_{x x} T_{x y}\right\rangle=\left\langle T_{x x} T_{y x}\right\rangle=\left\langle\sin \varphi(\mathbf{r}) \cos ^{3} \varphi(\mathbf{r})\right\rangle=0$ \\
\hline$\left\langle T_{x y} T_{y y}\right\rangle=\left\langle T_{y x} T_{y y}\right\rangle=\left\langle\sin ^{3} \varphi(\mathbf{r}) \cos \varphi(\mathbf{r})\right\rangle=0$ & $\left\langle T_{x x} T_{y y}\right\rangle=\left\langle\sin ^{2} \varphi(\mathbf{r}) \cos ^{2} \varphi(\mathbf{r})\right\rangle=1 / 8$ \\
\hline
\end{tabular}

Table 1. First-order and Second-order moments of the elements in Jones matrix

In this case, the coherence matrix $\mathbf{W}^{t}$ is easily found by substituting Eqs. (22-(23) into Eq (20), with the result 


$$
\mathbf{W}^{t}\left(\mathbf{r}_{1}, \mathbf{r}_{2}\right)=\frac{1}{4}\left(\begin{array}{ll}
W_{x x}^{i} & W_{x y}^{i} \\
W_{y x}^{i} & W_{y y}^{i}
\end{array}\right)+\frac{1}{8} \wedge\left(\frac{\Delta r_{x}}{l}\right) \wedge\left(\frac{\Delta r_{y}}{l}\right)\left(\begin{array}{cc}
W_{x x}^{i}+W_{y y}^{i} & -W_{x y}^{i}+W_{y x}^{i} \\
-W_{y x}^{i}+W_{x y}^{i} & W_{y y}^{i}+W_{x x}^{i}
\end{array}\right) .
$$

Equation (24) provides us with the desired correlation properties of the modulated electric field passing through the depolarizer of the random polarizer array. For demonstration purpose only, and without loss of generality, we will perform our calculations only for an incident field that is a linearly polarized, spatially coherent Gaussian beam with the electric field making an angle $\theta$ with the $\hat{x}$ axis. On substituting into Eq. (24), the expression for the mutual coherence matrix of the modulated beam just behind the depolarizer is

$$
\mathbf{W}^{t}\left(\mathbf{r}_{1}, \mathbf{r}_{2}\right)=\frac{I_{o}}{8} \exp \left\{-\frac{\left|\mathbf{r}_{1}\right|^{2}+\left|\mathbf{r}_{2}\right|^{2}}{r_{s}^{2}}\right\} \times\left\{2\left(\begin{array}{cc}
\cos ^{2} \theta & \sin \theta \cos \theta \\
\sin \theta \cos \theta & \sin ^{2} \theta
\end{array}\right)+\wedge\left(\frac{\Delta r_{x}}{l}\right) \wedge\left(\frac{\Delta r_{y}}{l}\right)\left(\begin{array}{ll}
1 & 0 \\
0 & 1
\end{array}\right)\right\} .
$$

where $I_{o}$ and $r_{s}$ are the on-axis intensity and the beam width of the incident field, respectively. Our interest in this demonstration is the statistical properties of the polarization speckle generated from the depolarizer. After replacements of A, B and D in Eq. (14) by the corresponding matrix elements in Eq. (15), we obtain the Green's function for free space propagation. After substituting of Eq. (25) into Eq.(13), one can calculate the free space propagation of the mutual coherence matrix and study the spatial evolutions of the degree of polarization and the degree of coherence in any observation plane $z>0$ in the case where the random polarizer array is illuminated by a linearly polarized Gaussian electromagnetic beam. To present some numerical examples, we have taken the following parameters: $r_{s}=1 \mathrm{~mm}, l=0.1 \mathrm{~mm}, \theta=\pi / 4$ and $\lambda=0.633 \mu \mathrm{m}$. Figure 6(a) gives the absolute values of the degree of spatial coherence of the polarization speckle at two positions $\Delta \mathbf{p}=\mathbf{p}_{1}-\mathbf{p}_{2}$ located symmetrically with respect to the $\hat{z}$ axis along the normalized propagation distance $z / z_{R}$. It can readily be seen that the degree of spatial coherence takes a large value close to unity for two points located near the optical axis. Fig. 6(b) shows the spatial degree of polarization of the electric field modulated by the depolarizer for free-space propagation, plotted against the normalized propagation distance $z / z_{R}$ and normalized lateral distance measured in spot size $p / r_{s}$. As expected, the spatial degree of polarization changes appreciably depending both on the propagation distance $z$ and the observation position $\mathbf{p}$. These figures indicate how the spatial degree of polarization and the degree of spatial coherence evolve for the selected polarization angle of the incident electric field.

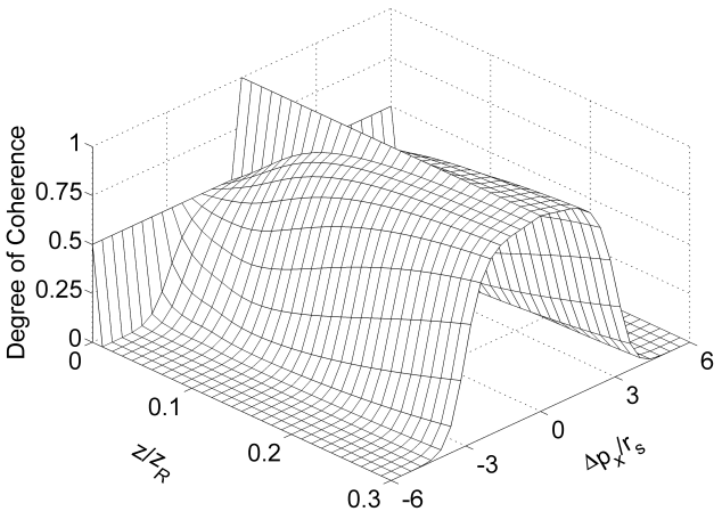

(a)

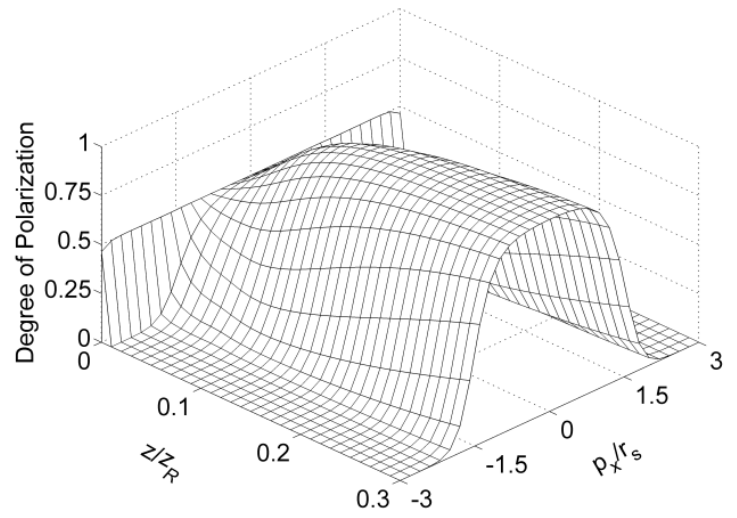

(b)

Figure 6 Degree of Coherence (a) and spatial degree polarization (b) for polarization speckle generated by random polarizer array

\section{CONCLUSIONS}

In summary, we review our recent work on the statistical properties of polarization speckle with its unique properties of random polarization states fluctuating in space. Based on the random polarization phasor sum as an extension of conventional random phasor sum, the first and second moments of the Stokes parameters of the resultant polarization speckle have been examined to depict its generation and development. After introduction of two types of depolarizers, i.e. a rough-surfaced retardation plate and a random polarizer array, we provide the origins of the polarization speckle from polarization interference by considering the vector nature for stochastic electric fields. Some statistical properties of the stochastic electric fields generated from these two depolarizers are reviewed in order to understand the performance of these two polarization optics devices for generation and development of the polarization speckle. 


\section{ACKNOWLEDGMENTS}

We are thankful to the following people with whom we worked and part of our joint work is introduced in this review:

Ning Ma, Chunmei Zhang, Sean Horder with Heriot-Watt University (UK) and Jonas Ritter, Wolfgang Osten with University of Stuttgart (Germany).

\section{REFERENCES}

[1] Dainty, J. C., Ed., [Laser Speckle and Related Phenomena], Springer-Verlag (1984).

[2] Goodman, J. W., [Statistical Optics], Wiley-Interscience, New York (2000).

[3] Goodman, J. W., [Speckle phenomena in Optics: Theory and Applications], Roberts-Company, Colorado (2006).

[4] Wolf, E., [Introduction to the theory of coherence and polarization of light], Cambridge University Press, New York (2007).

[5] Zhang, S., Takeda, M., and Wang, W., "Statistics of spatial derivatives of Stokes parameters for isotropic random polarization field," JOSA A27, 1999-2003 (2010).

[6] Ma, N., Hanson, S. G., Takeda, M., and Wang, W., "Coherence and polarization of polarization speckle generated by arough-surfaced retardation plate depolarizer," JOSA A 322346-2352 (2015).

[7] Ritter, J., Ma. N., Osten, W., Takeda, M., and Wang, W., "Depolarizing surface scattering by a birefringent material with rough surface," Opt. Comm. 430 456-460 (2019).

[8] Siegman, A. E., [Lasers] University Science, 1986.

[9] Yura, H. T. and Hanson, S. G. "Optical beam wave propagation through complex optical systems," JOSA. A 4, 1931-1948 (1987).

[10] Hecht, E., [Optics] Addison-Wesley, (2015).

[11] Wang, W., Matsuda, A., Hanson, S. G., Takeda, M., "The spatial degree of polarization and the first-order statistical properties of polarization speckle," Proc. SPIE 7063, 70630B (2008).

[12] Takeda, M., Wang. W., Naik, D. N. and Singh. R. K., "Spatial statistical optics and spatial correlation holography: a review," Opt. Rev. 21, 849-861 (2014).

[13] Ma, N., Hanson, S. G., and Wang, W., "Modulation of electromagnetic fields by a depolarizer ofrandom polarizer array," Opt. Lett (41) 1997-2000 (2016).

[14] Mandel, L. and Wolf, E. [Optical Coherence and Quantum Optics], Cambridge Univ. Press (1995).

[15] O'Neill, E. L. [Introduction to Statistical Optics] Addison-Wesley, (1965). 